ITP-UH-07/94

April 1994

cond-mat/9405038

\title{
NEW FAMILY OF SOLVABLE 1D HEISENBERG MODELS
}

\author{
HOLGER FRAHM⿵⺆ \\ Institut für Theoretische Physik, Universität Hannover, D-30167 Hannover, Germany \\ VLADIMIR I. INOZEMTSEV巾 \\ Laboratory of Theoretical Physics, JINR, 141980 Dubna, Russia
}

\begin{abstract}
Starting from a Calogero-Sutherland model with hyperbolic interaction confined by an external field with Morse potential we construct a Heisenberg spin chain with exchange interaction $\propto 1 / \sinh ^{2} x$ on a lattice given in terms of the zeroes of Laguerre polynomials. Varying the strength of the Morse potential the HaldaneShastry and harmonic spin chains are reproduced. The spectrum of the models in this class is found to be that of a classical one-dimensional Ising chain with nonuniform nearest neighbour coupling in a nonuniform magnetic field which allows to study the thermodynamics in the limit of infinite chains.
\end{abstract}

PACS-numbers: $75.10 . J m$ 05.30.-d $05.50 .+\mathrm{q}$

\footnotetext{
*e-mail: frahm@itp.uni-hannover.de

${ }^{\dagger}$ e-mail: inozv@theor.jinrc.dubna.su
} 
Studies of Heisenberg spin chains with exchange coupling proportional to certain functions decaying like the inverse square of the distance have unveiled surprisingly simple properties which allow for a detailed study of these many-body systems. Among of these properties are exact wave functions of Jastrow product form and very simple spectra. Most notably, the Haldane-Shastry model [1, 2] with trigonometric $\left(\propto 1 / \sin ^{2} x\right)$ exchange admits an interpretation as the generalization of the concept of an "ideal gas" to the case of fractional statistics [3, 4, 5].

All of these models can be related to Calogero-Sutherland models of particles with an internal degree of freedom (spin) using the exchange operator formalism [6]. Within this approach, the spin chains are obtained by "freezing out" the kinematic degrees of freedom in these models thereby giving a pure exchange model of particles interacting with inverse square potential on a lattice given by the static equilibrium positions of the Calogero model. Within this formalism it has been possible to construct a family of operators commuting with the Hamiltonian and among themselves for the Haldane-Shastry model [7] and a model with rational exchange related to the $1 / x^{2}$ Calogero model confined by a harmonic potential well [8, 9, 10] (due to its spectrum this model will be referenced as the "harmonic spin chain" in the following). Although it has not been possible so far to obtain the Hamiltonian from this construction directly this is to be considered as the proof of integrability of these models.

In the limit of infinite chains the above considerations have been applied to the hyperbolic chain with exchange $\propto 1 / \sinh ^{2} x$ on a translationally invariant lattice. A generalization of this case to finite nonuniform lattices which reduces to the Haldane-Shastry and the harmonic spin chain in certain limits is presented in this letter. We start with the Hamiltonian of a $N$-particle Calogero-Sutherland system of particles interacting with an external field with Morse potential given by

$$
\mathcal{H}_{C S}=\sum_{j}^{N}\left[\frac{p_{j}^{2}}{2}+2 \tau^{2}\left(e^{4 x_{j}}-2 e^{2 x_{j}}\right)\right]+\sum_{j<k}^{N} \frac{1}{\sinh ^{2}\left(x_{j}-x_{k}\right)} .
$$

The classical equilibrium positions of the particles (that will later define the lattice on which the spins are placed) are given in terms of the set of nonlinear algebraic equations

$$
-\sum_{k \neq j}^{N} \frac{z_{k}\left(z_{j}+z_{k}\right)}{\left(z_{j}-z_{k}\right)^{3}}+\tau^{2}\left(z_{j}-1\right)=0(j=1, \ldots, N),
$$

where we have introduced $z_{j}=e^{2 x_{j}}$. A general solution to (2) is unknown. However, following the observation by Calogero [11] for $1 / x^{2}$ case we assume that the $\left\{z_{j}\right\}$ are the roots of some polynomial $p_{N}(z)=\prod_{j=1}^{N}\left(z-z_{j}\right)$ obeying the second order differential equation [12]

$$
A p_{N}^{\prime \prime}+B p_{N}^{\prime}+\lambda_{N} p_{N}=0
$$


where $A$ and $B$ are polynomials in $z$ and $\lambda_{N}$ is some constant. Choosing $\lambda_{N}=2 N \tau$ we find that (3) reads

$$
y \frac{d^{2} p_{N}(y)}{d y^{2}}+(-y+1+\Gamma) \frac{d p_{N}(y)}{d y}+N p_{N}(y)=0, \quad y=2 \tau z
$$

with $\Gamma=2(\tau-N)+1$ from which we identify $p_{N}$ as the Laguerre polynomial $L_{N}^{(\Gamma)}(2 \tau z)$. Let us note the following properties of $L_{N}^{(\Gamma)}$ :

1. For $\Gamma>-1$ all roots of $L_{N}^{(\Gamma)}$ are real positive numbers.

2. $L_{N}^{(\Gamma)}$ has multiple zeroes if and only if $\Gamma=-n, n=2,3, \ldots, N$. At these values of $\Gamma$ one can write

$$
L_{N}^{(-n)}(y)=\frac{(N-n) !}{N !}(-1)^{(n)} y^{n} L_{N-n}^{(n)}(y)
$$

3. As $\Gamma=-n+\varepsilon, \varepsilon \rightarrow 0$ the $n$ roots of $L_{N}^{(\Gamma)}$ approaching 0 have the following asymptotic behaviour:

$$
z_{j} \sim \text { const }|\varepsilon|^{\frac{1}{n}} \exp \left(\frac{2 \pi i j}{n}\right), \quad j=1, \ldots, n
$$

Introducing variables $z_{j}=1+\tau^{-\frac{1}{2}} \zeta_{j}$ and taking the limit of large $\tau$ the rational limit of the Calogero model related to the harmonic spin chain is obtained. In this case the lattice points $\zeta_{j}$ are the roots of the Hermite polynomial $H_{N}(\zeta)$. For the special values of $\Gamma$ being a negative integer $\geq-N$ the lattice separates into two parts not coupled by the interaction term in the Hamiltonian. Upon rescaling one of them is given by $n$-th roots of unity (5), which results into a lattice of equally spaced sites with periodic boundary conditions. The corresponding spin chain is the Haldane-Shastry model with interaction $\propto 1 / \sin ^{2} x$. The other part corresponds to the hyperbolic model with $\Gamma$ replaced by $-\Gamma$. Finally, as $\Gamma \rightarrow-N$ the model reduces to the trigonometric model.

Following Fowler and Minahan [7] we now consider the a model where $N$ bosonic particles are sitting on different points $z_{j}$ satisfying (2) and allow the exchange of particle positions as the only dynamical process. Denoting the corresponding Hermitian exchange operator for particles $i$ and $j$ by $\mathcal{M}_{i j}$ we choose the Hamiltonian of this system to be

$$
\mathcal{H}_{e x}=\sum_{j<k}^{N} h_{j k} \mathcal{M}_{j k}, \quad h_{j k}=\frac{z_{j} z_{k}}{\left(z_{j}-z_{k}\right)^{2}} .
$$

We now construct operators

$$
h_{j}=\frac{1}{4}\left(\hat{\pi}_{j}+\omega_{j}\right)\left(\hat{\pi}_{j}-\omega_{j}\right)-\tau^{2}\left(z_{j}-1\right)^{2}+\text { const } \omega_{j}
$$


where

$$
\hat{\pi}_{j}=\sum_{k \neq j}^{N} \frac{z_{j}+z_{k}}{z_{j}-z_{k}} \mathcal{M}_{j k}, \quad \omega_{j}=\sum_{k \neq j}^{N} \mathcal{M}_{j k}
$$

At some value of the constant in (7) these operators can be factorized:

$$
h_{j}=a_{j}^{+} a_{j}^{-}, \quad a_{j}^{ \pm}=\frac{1}{2}\left(\hat{\pi}_{j} \pm \omega_{j}\right) \pm \delta \tau\left(z_{j}-1\right), \quad \delta^{2}=1 .
$$

Commutators of two operators from this set are a bit more complicated than in the case of harmonic chain,

$$
\left[h_{j}, h_{k}\right]=2 \delta \tau\left(h_{j} \mathcal{M}_{j k}-\mathcal{M}_{j k} h_{j}\right)+\omega_{j} \mathcal{M}_{j k} h_{j}-h_{j} \mathcal{M}_{j k} \omega_{j}
$$

Using the properties

$$
\begin{gathered}
\mathcal{M}_{i j} z_{i}=z_{j} \mathcal{M}_{i j}, \quad \mathcal{M}_{i j} z_{k}=z_{k} \mathcal{M}_{i j} \quad \text { for } i \neq k \neq j \\
\mathcal{M}_{i j k}=\mathcal{M}_{i k} \mathcal{M}_{i j}=\mathcal{M}_{i j} \mathcal{M}_{j k}=\mathcal{M}_{j k} \mathcal{M}_{i k}
\end{gathered}
$$

of the exchange operators $\mathcal{M}_{i j}$ one finds that the commutators $\left[h_{j}, \mathcal{H}_{e x}\right]=0$ for an arbitrary set of solutions to (2) and hence the operators

$$
\mathcal{I}_{m}=\sum_{j=1}^{N} h_{j}^{m}
$$

all commute with the Hamiltonian (6). For the Haldane-Shastry model [7] and the harmonic spin chain [8] it can be shown that operators similar to these $\mathcal{I}_{m}$ also commute among themselves. We have not been able to prove this in the general case considered here. After tedious calculations we were only able to show that $\left[\mathcal{I}_{n}, \mathcal{I}_{m}\right]=0$ for $1 \leq n, m \leq 3$.

As usual the operators $\mathcal{M}_{j k}$ can be related to exchange operators acting not on the particle positions but on their internal degree of freedom as they are defined in the space of bosonic, i.e. totally symmetric wavefunctions. This leads us to consider the Hamiltonian

$$
\mathcal{H}=-\sum_{j<k}^{N} h_{j k} \frac{\vec{\sigma}_{j} \cdot \vec{\sigma}_{k}-1}{2}=C_{N}-\sum_{j<k}^{N} h_{j k} \mathcal{P}_{j k}, \quad C_{N}=\frac{1}{24} N(N-1)(3 \Gamma+2 N-1)
$$

with the spin exchange operator $\mathcal{P}_{j k}=\frac{1}{2}\left(\vec{\sigma}_{j} \cdot \vec{\sigma}_{k}-1\right)$. The $\sigma_{j}^{\alpha}$ are Pauli matrices acting on the $j^{\text {th }}$ site of the lattice. The same procedure gives a trivial exchange operator $\tilde{\mathcal{I}}_{1}=-\frac{1}{3} \sum \mathcal{P}_{i j k}$ for the spin analogue of $\mathcal{I}_{1}$. The next operator in this sequence, $\tilde{\mathcal{I}}_{2}$, already contains four-spin exchange terms with nonuniform exchange couplings.

To construct eigenstates of the Hamiltonian (10) we start from the ferromagnetic vacuum $|0\rangle=|\uparrow \uparrow \ldots \uparrow\rangle$ and consider states with given magnetization

$$
\left|\psi^{(M)}\right\rangle=\sum_{j_{1}<\ldots<j_{M}}^{N} \psi\left(j_{1} \ldots j_{M}\right) \prod_{s=1}^{M} \sigma_{s}^{-}|0\rangle .
$$


Using the fact that the $z_{j}$ are roots of the $N$-th Laguerre polynomial we find that the eigenstates in the one-magnon sector $(M=1)$ have amplitudes

$$
\psi_{m}(j) \propto z_{j}^{m} \frac{L_{N-m-1}^{(\Gamma+2 m)}\left(2 \tau z_{j}\right)}{L_{N-1}^{(\Gamma)}\left(2 \tau z_{j}\right)}, \quad m=0, \ldots, N-1 .
$$

Their energies $E_{m}^{(1)}=\epsilon_{m}$ are given by the following quadratic dispersion law:

$$
\epsilon_{m}=\frac{m}{2}(\Gamma+m)
$$

Next we have studied the two-magnon sector $(M=2)$. The amplitudes $\psi\left(j_{1}, j_{2}\right)$ can be written as polynomials in $\left\{z_{j}^{-1}\right\}$. No closed expression for these amplitudes has been found. Nevertheless, the eigenproblem can be solved analytically and we were able to find the complete set of $N(N-1) / 2$ eigenvalues which can be written as

$$
E_{m, n}^{(2)}=\epsilon_{m}+\epsilon_{n}\left(1-\delta_{m, n-1}\right), \quad 0 \leq m<n \leq N-1
$$

with the single magnon energies $\epsilon_{m}$ given by (13).

Finally we considered the $M$-magnon sector. Within the Ansatz

$$
\psi=\frac{\prod_{\lambda>\mu}^{M}\left(z_{j_{\lambda}}-z_{j_{\mu}}\right)^{2} F\left(z_{j_{1}} \ldots z_{j_{M}}\right)}{\prod_{\nu=1}^{M} z_{j_{\nu}} p_{N}^{\prime}\left(z_{j_{\nu}}\right)}
$$

where $F$ is some symmetric polynomial in $\{z\}$, the corresponding part of spectrum which comprises $\frac{(N-M+1) !}{M !(N-2 M+1) !}$ eigenvalues is found to be additive

$$
E_{\left\{m_{k}\right\}}^{(M)}=\sum_{k=1}^{M} \epsilon_{m_{k}}
$$

with the dispersion (13) where the integers $m_{k}$ obey the set of restrictions

$$
0 \leq m_{1}<m_{2}-1<\ldots<m_{M}-1 \leq N-2
$$

While our solution of the $M$-particle sector is not complete we make the following hypothesis concerning the spectrum of the class of models ([10):

All the eigenvalues of $\mathcal{H}$ can be written in compact form

$$
E_{n_{1} \ldots n_{N}}=\sum_{k=1}^{N-1} \epsilon_{k} n_{k+1}\left(1-n_{k}\right)
$$

where $\epsilon_{k}=\frac{1}{2} k(\Gamma+k)$ and $\left\{n_{k}\right\}=0,1$. As a consequence, the operator $\mathcal{H}=-1 / 2 \sum_{j<k}^{N} h_{j k} \vec{\sigma}_{j} \vec{\sigma}_{k}$ is equivalent up to unitary transformation to the Hamiltonian of classical 1D Ising chain in a 
non-uniform magnetic field

$$
\begin{aligned}
\mathcal{H}_{I} & =\sum_{k=1}^{N-1} \epsilon_{k}\left(\sigma_{k+1}-\sigma_{k}-\sigma_{k+1} \sigma_{k}\right) \\
& =\epsilon_{N-1} \sigma_{N}+\sum_{k=0}^{N-2}\left[\sigma_{k+1}\left(\epsilon_{k}-\epsilon_{k+1}\right)-\sigma_{k+1} \sigma_{k+2} \varepsilon_{k+1}\right]
\end{aligned}
$$

with $\left\{\sigma_{k}\right\}= \pm 1$.

We have checked this hypothesis numerically to give the correct spectrum for lattices of lengths up to $N=12$. We have also confirmed it in the limit $\Gamma \rightarrow \infty$ by establishing the analytic correspondence to the effective Hamiltonian of the harmonic chain (Eq. (20) in [9]). Finally, it is consistent with the picture of lattice separation at $-N \leq \Gamma=-n<-1$ (where $\left.\epsilon_{n}=0\right)$ mentioned above and coincides with the Ising Hamiltonian that follows for the HaldaneShastry model in the appropriate limit of the Hubbard model with $1 / r$ hopping [13.

The simple form of the spectrum (18) allows to compute the free energy in the thermodynamic limit by using the transfer matrix method. The partition function on a finite lattice can be written as

$$
\begin{aligned}
\mathcal{Z}_{N}=\frac{1}{2} \operatorname{trace}\left\{\left(\begin{array}{cc}
1 / w_{1} & -1 / w_{1} \\
1 & 1
\end{array}\right)\right. & \prod_{k=1}^{N-2}\left[\left(\begin{array}{cc}
1+w_{k} & 0 \\
0 & 1-w_{k}
\end{array}\right) \mathcal{B}_{k}\right] \times \\
& \left.\times\left(\begin{array}{cc}
\left(1+w_{N-1}\right)^{2} & \left(1+w_{N-1}\right)^{2} \\
\left(1-w_{N-1}\right)^{2} & \left(1-w_{N-1}\right)^{2}
\end{array}\right)\right\}
\end{aligned}
$$

where

$$
\mathcal{B}_{k}=\frac{1}{2}\left(\begin{array}{cc}
1+\frac{w_{k}}{w_{k+1}} & 1-\frac{w_{k}}{w_{k+1}} \\
1-\frac{w_{k}}{w_{k+1}} & 1+\frac{w_{k}}{w_{k+1}}
\end{array}\right), \quad w_{k}=e^{-\frac{1}{2} \beta \epsilon_{k}}
$$

To perform the thermodynamic limit $N \rightarrow \infty$ we rescale the magnon energies (13) with a factor $1 / N^{2}$ and find the leading term of (19) to be $Z \sim \exp \left(\beta E_{0}\right) \prod_{k=1}^{N-2}\left(1+w_{k}\right)$ with some proper $E_{0}$ renormalizing the ground state energy to zero. From this expression one obtains for the free energy per site

$$
f=-\frac{E_{0}}{N}-\frac{1}{\beta} \int_{0}^{1} d x \ln \left(1+e^{-\beta \epsilon(x) / 2}\right) .
$$

Using the quasiparticle dispersion $\epsilon(x)=x(\gamma+x)$ (the renormalization of the magnon energies leads to $\gamma=\Gamma / N$ when taking the thermodynamic limit of (13)) this can be written for $-1<\gamma<0$ as

$$
f=-\frac{1}{\beta}\left(\int_{0}^{-\gamma} d x \ln \left(1+e^{\beta \epsilon(x) / 2}\right)+\int_{-\gamma}^{1} d x \ln \left(1+e^{-\beta \epsilon(x) / 2}\right)\right)
$$


Note that changing the sign of the quasiparticle dispersion or, equivalently, the exchange in (10) from ferromagnetic to antiferromagnetic changes the free energy by a temperature independent term (due to the different ground state energy) only. This has been noticed in the case of the Haldane-Shastry model $\gamma=-1$ before [3] and is a consequence of the possibility to describe these models in terms of an effective Ising model (18).

Since the total spin of the effective Ising model is proportional to the $z$-component of the total spin in the original model one can also study the effect of an external magnetic field. For $\Gamma>-1$ the largest eigenvalue of (10) in the $M$-magnon sector is

$$
E_{\max }=\frac{1}{6} M\left(3(N-M)(N-M+\Gamma)+M^{2}-1\right) \quad \text { for } M \leq \frac{1}{2} N
$$

from which we conclude that the ground state magnetization of the antiferromagnetic chain in a magnetic field $h$ is given by $\mathcal{M}(h)=\frac{1}{4}\left(\sqrt{\gamma^{2}+8 h}-\gamma\right)$ for $h \leq h_{s}=\frac{1}{2}(\gamma+1)$. Beyond $h_{s}$ the magnetization is saturated at $\frac{1}{2}$. For finite temperatures the transfer matrix method yields

$$
\mathcal{M}(\beta, h)=\frac{1}{2} \int_{0}^{1} d x \frac{\sinh \beta h / 2}{\sqrt{\exp (-\beta \epsilon(x))+\sinh ^{2} \beta h / 2}} .
$$

In this letter we have constructed a new family of solvable Heisenberg spin chains. As in the previously known Haldane-Shastry and harmonic models their spectra can be given in terms of an effective Ising Hamiltonian. This mapping allows to study the thermodynamics of this models in detail. Generalization to other exchange type models such as interacting $S U(N)$ spins or electrons subject to a supersymmetric $t-J$ or Hubbard type Hamiltonian are easily constructed by considering different representations of the permutation operators in (10). At the same time, a number of interesting questions, especially regarding the construction of a complete set of conserved quantities, remains open.

This work has been partially supported by the Deutsche Forschungsgemeinschaft under grant no. Fr 737/2-1. One of the authors (V.I.) has received partial support within the framework of the Heisenberg-Landau program. 


\section{References}

[1] F. D. Haldane, Phys. Rev. Lett. 60, 635 (1988).

[2] B. S. Shastry, Phys. Rev. Lett. 60, 639 (1988).

[3] F. D. M. Haldane, Phys. Rev. Lett. 66, 1529 (1991).

[4] D. Bernard, V. Pasquier, and D. Serban, "A one dimensional ideal gas of spinons, or some exact results on the XXX spin chain with long range interaction", preprint SPhT-93-117.

[5] F. D. M. Haldane, "Physics of the ideal semion gas: Spinons and quantum symmetries of the integrable Haldane-Shastry spin chain", in Proceedings of the 16th Taniguchi Symposium, Kashikojima, Japan, October 1993, edited by A. Okiji and N. Kawakami, Berlin, 1994, Springer.

[6] A. P. Polychronakos, Phys. Rev. Lett. 69, 703 (1992).

[7] M. Fowler and J. A. Minahan, Phys. Rev. Lett. 70, 2325 (1993).

[8] A. P. Polychronakos, Phys. Rev. Lett. 70, 2329 (1993).

[9] H. Frahm, J. Phys. A 26, L473 (1993).

[10] A. P. Polychronakos, "Exact spectrum of $S U(N)$ spin chain with inverse-square exchange", preprint CERN-TH-7040/93 (1993).

[11] F. Calogero, Lett. Nuovo Cimento 19, 505 (1977).

[12] V. I. Inozemtsev, Phys. Scripta 39, 289 (1989).

[13] F. Gebhard and A. E. Ruckenstein, Phys. Rev. Lett. 68, 244 (1992). 